OPEN ACCESS

Edited by:

Fangwei Liu,

China Medical University, China

Reviewed by:

Emilia Lecuona,

Northwestern University,

United States

Laura Andrea Dada,

Northwestern University,

United States

${ }^{*}$ Correspondence:

Quan Li

quanligene@126.com

Li-Ming Zhang

ZhangL1@anes.upmc.edu

${ }^{\dagger}$ These authors have contributed equally to this work and

share first authorship

Specialty section:

This article was submitted to

Inflammation,

a section of the journal

Frontiers in Immunology

Received: 12 April 2021

Accepted: 02 July 2021

Published: 19 July 2021

Citation:

Jin S, Ding $X$, Yang C, Li W, Deng $M$

Liao $H, L v X$, Pitt BR, Billiar TR,

Zhang L-M and Li Q (2021) Mechanical Ventilation Exacerbates Poly (I:C) Induced Acute Lung Injury:

Central Role for Caspase-11 and Gut-Lung Axis.

Front. Immunol. 12:693874. doi: 10.3389/fimmu.2021.693874

\section{Mechanical Ventilation Exacerbates Poly (I:C) Induced Acute Lung Injury: Central Role for Caspase-11 and Gut-Lung Axis}

\author{
Shuqing Jin ${ }^{1,2 \dagger}$, Xibing Ding ${ }^{3 \dagger}$, Chenxuan Yang ${ }^{2,4}$, Wenbo $\mathrm{Li}^{2}$, Meihong Deng ${ }^{5}$, \\ Hong Liao ${ }^{2}$, Xin Lv ${ }^{1}$, Bruce R. Pitt ${ }^{6}$, Timothy R. Billiar ${ }^{2}$, Li-Ming Zhang ${ }^{7 *}$ and Quan Li $^{8^{*}}$ \\ ${ }^{1}$ Department of Anesthesiology, Shanghai Pulmonary Hospital, TongJi University, Shanghai, China, ${ }^{2}$ Department of Surgery, \\ University of Pittsburgh Medical School, Pennsylvania, PA, United States, ${ }^{3}$ Department of Anesthesiology, Renii Hospital, \\ Shanghai Jiaotong University Medical School, Shanghai, China, ${ }^{4}$ Department of Breast Surgical Oncology, National Cancer \\ Center/National Clinical Research Center for Cancer/Cancer Hospital, Chinese Academy of Medical Sciences and Peking \\ Union Medical College, Beijing, China, ${ }^{5}$ Department of Surgery, The Ohio State University, Ohio, OH, United States, \\ ${ }^{6}$ Department of Environmental Occupational Health, University of Pittsburgh Graduate School Public Health, Pennsylvania, \\ PA, United States, ${ }^{7}$ Department of Anesthesiology and Perioperative Medicine, University of Pittsburgh School of Medicine, \\ Pennsylvania, PA, United States, ${ }^{8}$ Department of Anesthesiology, National Cancer Center/National Clinical Research Center \\ for Cancer/Cancer Hospital \& Shenzhen Hospital, Chinese Academy of Medical Sciences and Peking Union Medical College, \\ Shenzhen, China
}

Background: The mechanisms by which moderate tidal volume ventilation (MTV) exacerbates preexisting lung injury are unclear. We hypothesized that systemic endotoxemia via the gut-lung axis would lead to non-canonical and canonical inflammasome activation and pyroptosis in a two-hit model involving polyinosinicpolycytidylic acid (Poly(l:C)), a synthetic analog of dsRNA and MTV and that this would associate with acute lung injury (ALI).

Methods: Anesthetized mice were administered Poly $(\mathrm{l}: \mathrm{C})$ intratracheally and then $6 \mathrm{~h}$ later, they were mechanically ventilated for $4 \mathrm{~h}$ with otherwise non-injurious MTV $(10 \mathrm{ml} /$ $\mathrm{kg})$. Changes in intestinal and alveolar capillary permeability were measured. Further documentation of ALI was assessed by evans blue albumin permeability, protein and IL-1 family concentration in bronchoalveolar lavage fluid (BALF) or plasma, and histopathology

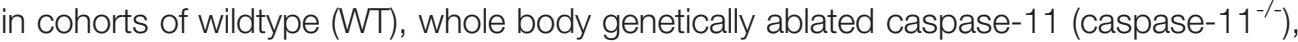

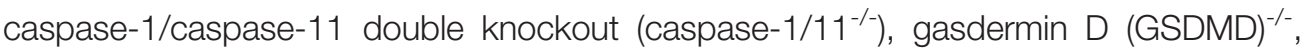
nucleotide-binding domain leucine-rich repeat-containing protein $3(\mathrm{NLRP} 3)^{-/-}$and advanced glycosylation end product-specific receptor (RAGE) ${ }^{-/-}$mice.

Results: Non-injurious MTV exacerbated the mild lung injury associated with Poly(l:C) administration. This included the disruption of alveolar-capillary barrier and increased levels of interleukin (IL)-6, high mobility group proteins 1 (HMGB-1), IL-1 $\beta$ in BALF and IL18 in plasma. Combined (Poly(I:C)-MTV) injury was associated with increase in gastrointestinal permeability and endotoxin in plasma and BALF. Poly(l:C)-MTV injury was sensitive to caspase-11 deletion with no further contribution of caspase-1 except for maturation and release of IL-18 (that itself was sensitive to deletion of NLRP3). Combined 
injury led to large increases in caspase-1 and caspase-11. Genetic ablation of GSDMD attenuated alveolar-capillary disruption and release of cytokines in combined injury model.

Conclusions: The previously noted exacerbation of mild Poly(l:C)-induced ALI by otherwise non-injurious MTV is associated with an increase in gut permeability resulting in systemic endotoxemia. The gut-lung axis resulted in activation of pulmonary noncanonical (cytosolic mediated caspase-11 activation) and canonical (caspase-1) inflammasome (NLRP3) mediated ALI in this two-hit model resulting in GSDMD sensitive alveolar capillary barrier disruption, pyroptosis (alveolar macrophages) and cytokine maturation and release (IL-1 $\beta ; \mathrm{IL}-18)$. Pharmacologic strategies aimed at disrupting communication between gut and lung, inhibition of inflammasomes or GSDMD in pyroptosis may be useful in ALI.

Keywords: acute lung injury, Poly(l:C), mechanical ventilation, gut-lung axis, systemic endotoxemia, caspase-11, caspase-1, pyroptosis

\section{INTRODUCTION}

Sepsis is the major underlying cause ( $75 \%)$ of acute respiratory distress syndrome (ARDS) and this often follows the onset of pneumonia (1). ARDS also occurs in a large number of patients from infections outside the lung and the mechanisms underlying the development of lung injury from remote sources are multifactorial and poorly understood (2). Both direct (e.g. pneumonia) and indirect (extrapulmonary) sepsis routinely require mechanical ventilation and it is well known that such lifesaving therapy can exacerbate underlying lung injury in an iatrogenic pathology of ventilator induced lung injury [VILI; (3)]. Indeed, minimizing over distension (volutrauma) and/or alveolar collapse and reopening (atelectrauma) by lungprotective ventilation (4) has had an impact in reducing morbidity and mortality from ARDS.

In preclinical studies, sensitization of VILI to preexisting acute lung injury (ALI) secondary to pneumonia $(5,6)$, intratracheal endotoxin (7-10), viral (11) and sterile injury (12-14) is apparent. Although preclinical outcomes vary as a function of magnitude of extrapulmonary septic condition and the nature of mechanical ventilation parameters [tidal volume, onset and duration; positive end expiratory pressure; (15)], sensitization of VILI to events originating in distal site and plasma space including exogenous endotoxin $(16,17)$ and polymicrobial sepsis $(18,19)$ has also been documented.

In the current study, we approached connections of direct and indirect lung injury that are predicted from possible gut-lung

Abbreviations: MTV, moderate tidal volume ventilation; Poly(I:C), polyinosinicpolycytidylic acid; ALI, acute lung injury; BALF, bronchoalveolar lavage fluid; WT, wild type; GSDMD, gasdermin D; NLRP3, nucleotide-binding domain leucinerich repeat-containing protein 3; RAGE, advanced glycosylation end productspecific receptor; IL, interleukin; HMGB1, high-mobility group box 1; TLR, toll like receptor; ARDS, acute respiratory distress syndrome; VILI, ventilator induced lung injury; LPS, lipopolysaccharide; FITC-D, fluoresceine isothiocyanate dextran; AMs, alveolar macrophages; PBS, phosphate buffered saline; RPMI, roswell park memorial institute; EBA, evans blue albumin; SEM, standard error of mean; H\&E, Hematoxylin \& Eosin; ASC, apoptosis-associated speck-like protein; CARD, caspase activation and recruitment domain; TNF, tumor necrosis factor. interactions and sensitization to subsequent non-injurious moderate mechanical tidal volume ventilation (MTV). We (20) and others (21) have shown that MTV can exacerbate lung injury after intratracheal delivery of polyinosinic-polycytidylic acid [Poly (I:C)], a synthetic analog of dsRNA (that itself can be produced by many viruses during their replicative cycle). The gut plays an important role in indirect lung injury by releasing infectious microbes and inflammatory, injurious mediators directly into the circulation or via the lymph system secondary to enhanced gut permeability (22). Endotoxin [lipopolysaccharide (LPS)] derived from gram negative microbes in the gut may thus be liberated in large amounts in the circulation and contribute to lung injury $(23,24)$. The canonical detection mechanism of LPS occurs via cell-surface toll like receptor-4 (TLR4), however but it is noteworthy that lung injury after Poly(I:C)-MTV (21) or systemic endotoxemia (25) involve TLR4 independent mechanisms. In this latter comprehensive study (25), the authors noted that indirect lung injury due to systemic endotoxemia involved non-canonical inflammasome caspase-11 mediated pyroptosis, an inflammatory programmed cell death, in pulmonary endothelium of intact mice. Murine caspase- 11 is the cytosolic receptor for LPS. Activation of caspase- 11 by LPS leads to cleavage of gasdermin D (GSDMD) and the $\mathrm{N}$-terminal cleavage fragment (GSDMD-N) leads to cell permeabilization and pyroptosis (26).

Accordingly, we: a) confirmed that pretreatment of intact mice with intratracheal (i.t.) Poly(I:C) would lead to sensitization to ALI due to otherwise non-injurious MTV; and b) hypothesized that ALI after Poly(I:C)-MTV was associated with gut derived LPS and caspase-11 non-canonical inflammasome mediated pyroptosis.

\section{MATERIALS AND METHODS}

\section{Experimental Protocols}

The Animal protocols were approved by the Animal Care and Use Committee and experiments were performed in strict 
adherence to NIH Guidelines and followed current guidelines for preclinical models in research. Protocols (with brief descriptions below) included: a) MTV enhanced Poly(I:C) induced ALI is associated with increased gastrointestinal permeability and increased endotoxin in plasma and lung; b) MTV exaggerates Poly(I:C) induced acute lung injury through a caspase-11 dependent process; and c) Regulation of caspase-11 expression and activation, its relationship to canonical nucleotide-binding domain leucine-rich repeat-containing protein 3 (NLRP3) mediated caspase- 1 activity and gasdermin cleavage dependent pathways in whole lung and isolated macrophages after Poly(I: C), MTV and their combination in intact mice.

\section{In-Vivo Experimental Animal Model}

C57 BL/6 mice (8-10 weeks old, male) were purchased from Jackson Laboratories. Caspase-1/11-/- mice, caspase-11-/- mice, TLR4-/- mice, NLRP3-/- mice, GSDMD-/- mice, advanced glycosylation end product-specific receptor (RAGE)-/- mice were bred and maintained in the University of Pittsburgh animal facility according to NIH animal care guidelines and all procedures were performed according to University of Pittsburgh Animal Research Protocols. A total number of 168 wild-type (WT) mice, 88 Caspase-1/11-/- mice, 88 Caspase-11-/mice, 32 NLRP3-/- mice, 40 GSDMD-/- mice, 16 RAGE-/- mice were used in this project.

The animal model protocols was as follows: Mice were prospectively randomized to one of four groups $(n=4-12$ per group): (a) SHAM: $100 \mu \mathrm{L}$ endotoxin free water intra-tracheal $10 \mathrm{~h}$ before the experimental endpoint with spontaneous breathing; (b) Poly(I:C): $3 \mathrm{mg} / \mathrm{kg}$ intra-tracheal Poly(I:C) (tlrlpicw, InvivoGen, USA), with spontaneous breathing for $10 \mathrm{~h}$; (c) MTV: $6 \mathrm{~h}$ after receiving a volume of $100 \mu \mathrm{L}$ intra-tracheal endotoxin free water then connected to a rodent ventilator, and ventilated for $4 \mathrm{~h}$ with tidal volume of $10 \mathrm{~mL} / \mathrm{kg}$, positive endexpiratory pressure of $0 \mathrm{~cm} \mathrm{H} \mathrm{H}_{2} \mathrm{O}, \mathrm{FiO}_{2} 0.21,140$ breaths/min; and (d) combined Poly(I:C)-MTV: $6 \mathrm{~h}$ after an intra-tracheal dose of $3 \mathrm{mg} / \mathrm{kg}$ Poly(I:C), mice were ventilated for $4 \mathrm{~h}$ before harvest. The ventilator parameters were the same of that in MTV group. In all groups, ketamine and xylazine were used to maintain anesthesia. Mean arterial blood pressure, heart rate and oxygen saturation were recorded using a mouse STARR system (Life Science Co.). Mice were sacrificed (10h after starting each protocol) by injecting peritoneal pentobarbitone $300 \mathrm{mg} / \mathrm{kg}$. Additional details were previously described (20).

Additional cohorts of wildtype, Caspase-1/11-/- and Caspase11-/- mice were prospectively randomized to same four groups as above. Lung tissue and freshly cultured primary alveolar macrophages were obtained for determination of pro- and cleaved caspases- 11 and -1 .

In addition, wildtype mice were prospectively randomized to these same four groups. Water bottles were removed from cages in the morning and $100 \mathrm{mg} / \mathrm{mL}$ FITC-D (4 kD) in PBS was administered ( $44 \mathrm{mg} / 100 \mathrm{~g}$ body weight) by oral gavage 4 hours before sacrifice. After 4 hours, anesthetize the mice by injecting peritoneal pentobarbitone and collect the blood using $1 \mathrm{ml}$ syringe with $25 \mathrm{G}$ needle by cardiac puncture. Blood or bronchoalveolar lavage fluid (BALF) was placed in microtainer tubes in the dark. Once blood and BALF have been collected from all the mice and all the samples was placed at $37^{\circ} \mathrm{C}$ for more than 1 hour, tubes were processed to centrifuge for 10 minutes at 800 grams. Concentration of fluoresceine isothiocyanate (FITC) in serum of BALF determined spectrophotofluorometrically (excitation $485 \mathrm{~nm}$; emission $528 \mathrm{~nm}$ ). A standard serially diluted FITC-Dextran (0 to $8 \mu \mathrm{g} / \mathrm{mL}$ ) was used. Serum from mice not injected with FITC-D was used as blank. In addition, endotoxin was measured (LAL Chromogenic endpoints assay, Hycult biotech, PA, USA) in serum and BALF of wildtype mice in these four groups.

An additional cohort of wildtype were assigned to four protocols above and at time of sacrifice, alveolar macrophages (AMs) and neutrophils, lymphocytes were obtained via bronchoalveolar lavage for short term culture and immunofluorescence staining. In brief, mice were bled by cardiac puncture and a catheter $(20 \mathrm{G})$ was inserted into tracheal and connected to $1 \mathrm{~mL}$ syringe filled with phosphate buffered saline [PBS $\left(\mathrm{Ca}^{2+} / \mathrm{Mg}^{2+}\right.$ free $\left.)\right]$. A total of $5 \mathrm{~mL}$ of PBS was used to wash lungs $(10 \mathrm{x})$ and the lavage fluid was centrifuged (600 g, 10 minutes) at $4^{\circ} \mathrm{C}$. The diluted cells were distributed on cell-counting plates and counted under a microscope. For differential cell sorting, cells were stained with Wright-Giemsa reagents (Baso, Zhuhai, China). The number of neutrophils, macrophages, and lymphocytes per 200 cells was determined based om morphology. Otherwise the diluted cells were resuspended in Roswell Park Memorial Institute [RPMI (2x10 cells $/ \mathrm{mL}$ )] with $12 \%$ serum and transferred to $35 \mathrm{~mm}$ petri dishes with $10 \mathrm{~mm}$ microwells (Mat Tek corp, Ashland, MA) and placed in incubator for $2 \mathrm{~h}$ to extract AMs. Media was changed and adherent cells (e.g. enriched in alveolar macrophages) were assessed for caspase-1 (FAM-FLICA ${ }^{\circledR}$ Caspase-1 Assay Kit (ImmunoChemistry Technologies, ImmunoChemistry Technologies, LLC), pyroptosis (In Situ Cell Death Detection Kit, TMR red (Sigma-Aldrich), and nuclear staining (Bisbenzimide Hoechst 33258) Imaging was observed and recorded with 600× magnification using a Olympus confocal microscope.

\section{Alveolar-Capillary Permeability}

Evans blue (Sigma-Aldrich) albumin (EBA; 0.5\%, $25 \mathrm{mg} / \mathrm{kg}$ ) was injected intravenously $1 \mathrm{~h}$ before euthanasia and lung harvesting. Blood samples and lung tissue were obtained and processed as described previously (18-20) and EBA permeability was calculated by dividing pulmonary EBA absorbance at $620 \mathrm{~nm} / \mathrm{g}$ lung tissue by plasma EBA absorbance at $620 \mathrm{~nm}$.

\section{Histological Examination}

For Hematoxylin \& Eosin (H \& E) staining, the left upper lobe was inflated with $4 \%$ paraformaldehyde, embedded in paraffin and assessed via semiquantitative histopathology at light microscopic level including following features: edema, hyperemia and congestion, neutrophil margination and tissue infiltration, intra-alveolar hemorrhage and debris, and cellular hyperplasia (18). Each feature was graded as absent, mild, moderate, or severe, with a score of 0-3. 


\section{Western Blot Analysis}

Cell lysis buffer (cell signaling technology) and a cocktail of protease inhibitors (Sigma-Aldrich) were used to extract protein in lung tissues and alveolar macrophages. $12 \%$ SDS gels was used for electrophoresis, Electrophoresis was performed at $80 \mathrm{~V}$ for 120 minutes. Then the protein in gels was transferred for 120 minutes at $200 \mathrm{~mA}$ to nitrocellulose membranes. 5\% milk in $1 \%$ Tween-20 in PBS was used to block membranes. The membranes were incubated with a primary antibody (anti-caspase-11 polyclonal antibody, 1:1000, abcam; anti-caspase-1 polyclonal antibody, 1:1000; abcam) at $4^{\circ} \mathrm{C}$ overnight and washed three times with PBST (0.1\% Tween-20 in PBS). Secondary antibody (1:5000; InvivoGen, USA) was then added and incubated at $37^{\circ} \mathrm{C}$ for $1 \mathrm{~h}$.

\section{Cytokines}

IL-1 $\beta$ (ELISA, R\&D), IL-6 (ELISA, R\&D), damage associated molecular pattern molecules (HMGB1, ELISA, Shino-test Corporation) concentrations in BALF and IL-18 in plasma (ELISA, Medical and Biological Laboratories CO., LTD) were determined by enzyme-linked immunosorbent assay (ELISA) according to the manufacturer's instructions.

\section{Statistical Analysis}

Statistical analysis was performed in GraphPad PRISM 7 (Graph Pad Software Inc.). All data were presented as the means \pm Standard Error of Mean (SEM). Data were analyzed by one-way analysis of variance (ANOVA) and Student-Newman-Keuls test if normally distributed. Mann-Whitney U-test was used for analyzing nonparametric data. In figures asterisks denote statistical significance $\left({ }^{*} p<0.05 ;{ }^{* *} p<0.01 ;{ }^{* *} p<0.001\right)$.

\section{RESULTS}

\section{MTV Enhanced Poly(I:C) Induced ALI Is Associated With Increased Gastrointestinal Permeability and Increased Endotoxin in Plasma and Lung}

Patients with severe respiratory viral infection may require ventilation and Poly(I:C) is a double stranded RNA immune stimulant used to mimic the immune activation of viral infections $(27,28)$. We (20) and others (21) have previously shown that Poly(I:C) pre-treatment markedly increases lung injury induced by moderate tidal volume ventilation (MTV=ventilation at $8-10 \mathrm{~mL} / \mathrm{kg}$ ). We assessed intestinal and alveolar-capillary permeability in Poly(I:C)-MTV mice by measuring the transmigration of FITC-D (4 kD) from gastrointestinal contents into plasma (Figure 1A) and BALF (Figure 1B) spaces, respectively. There were modest increases in both organs after either stimulus alone but the combination of 3 $\mathrm{mg} / \mathrm{kg}$ intra-tracheal Poly(I:C) for $6 \mathrm{~h}$ followed by MTV for $4 \mathrm{~h}$ led to large increases in both gastrointestinal permeability and alveolar-capillary permeability to FITC-D. We then measured endotoxin in plasma (Figure 1C) and BALF (Figure 1D) and noted small increases after either stimulus alone but large increases in endotoxin in plasma and BALF after combined Poly(I:C)-MTV. It is unlikely that endotoxin was due to contamination of Poly(I:C) as the solution tested negative prior to intratracheal instillation. Further refinement and quantitation of lung injury (EBA permeability, BALF protein, histology, cytokine release) was used to assess the nature of interaction of Poly(I:C) and MTV in Figure 2.

Increased levels of circulating and intrapulmonary endotoxin after combined injury prompted us to pursue potential role of the intracellular endotoxin receptor caspase-11 (TLR4-independent) in acute lung injury.

\section{MTV Exaggerates Poly(I:C) Induced Acute Lung Injury Through a Caspase-11 Dependent Process}

Viral infection can activate interferon responses and this can promote the up regulation of the caspase- 11 non-canonical inflammasome (27). To determine if up regulation of caspase11 in the lungs contributed to the pulmonary response to ventilation, we pre-treated wild-type and caspase-11-/- mice with Poly(I:C) followed $6 \mathrm{~h}$ later with MTV for $4 \mathrm{~h}$. Caspase11 activation can promote the activation of the caspase-1 canonical inflammasome $(29,30)$. Therefore, to determine the relative contribution of caspase-1 to the injury response we also included mice deficient in both caspase-1 and caspase-11. As shown in Figure 2 [and consistent with the above findings (Figure 1) and our previous findings (18-20)], MTV alone for $4 \mathrm{~h}$ had no impact on indices of lung injury including leakage of Evans blue dye into the lung (Figure 2A), accumulation of protein into the BAL fluid (BALF; Figure 2B), or histologic scoring of lung injury (Figures 2C, D). While Poly(I:C) treatment alone induced modest increases in Evans blue dye (Figure 2A) and protein accumulation (Figure 2B) in the BALF, the initiation of MTV at $6 \mathrm{~h}$ after Poly(I:C) markedly increased the appearance of these large molecular weight species as well as histopathologic quantitative assessment of nature of ALI (Figure 2D). The deletion of caspase-11 had no impact on the mild lung injury induced by $\operatorname{Poly}(\mathrm{I}: \mathrm{C})$ alone but almost completely prevented the exaggerated injury induced by the addition of MTV to Poly(I:C). No further protection was seen in mice deficient in both caspase-11 and caspase-1 consistent with caspase-11 being central to acute lung injury, induced by sequential hits.

To assess the requirement for caspase- 11 and caspase- 1 on inflammatory mediator production, IL-6, HMGB1 and IL-1 $\beta$ were measured in BALF (Figures 3A-C) and IL-18 in plasma (Figure 3D). Similar to the observations made on lung injury, the addition of MTV to Poly(I:C) significantly increased levels of IL6 , IL-1 $\beta$ and HMGB1 in the BALF and IL-18 in the plasma. Deletion of caspase-11 alone or caspase-11 and caspase- 1 together significantly suppressed the increases in these mediators induced by MTV+Poly(I:C). A significant difference in the degree of mediator suppression between the mouse strains was seen for BALF IL- $1 \beta$ levels and plasma IL-18 levels, where deletion of both caspase-11 and caspase-1 lead to an even greater decrease in IL$1 \beta$ and IL-18 levels than that seen with deletion of caspase-11 
A

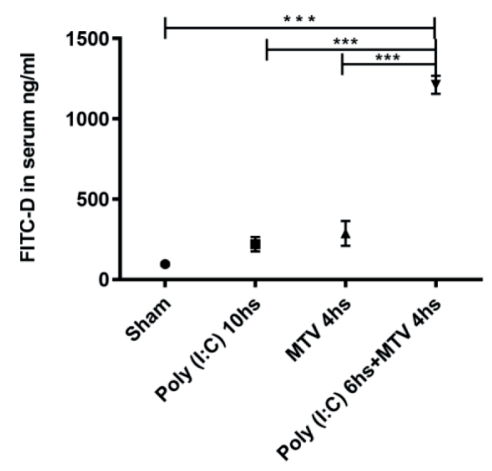

C

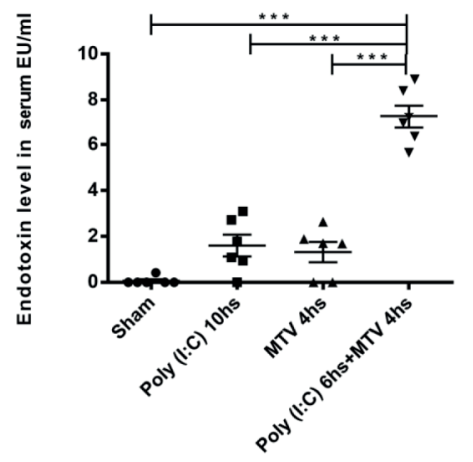

B

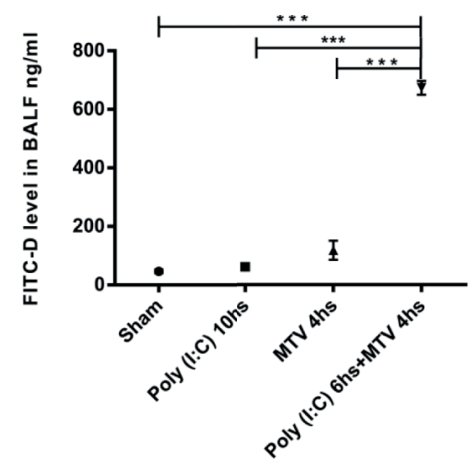

D

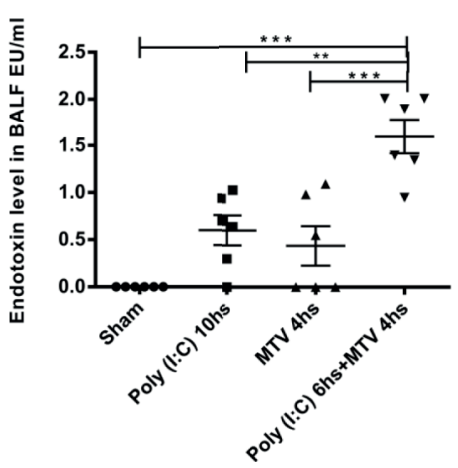

FIGURE 1 | Intestinal and alveolar-capillary permeability. Intestinal permeability of mice evaluated by FITC-D levels in serum (A) and in bronchoalveolar lavage fluid (BALF) (B) $200 \mu \mathrm{L}$ FITC-D (30 mg/mL) was instilled through orogastric feeding. Endotoxin levels in serum (C) and in BALF (D) were measured. All PBS and Poly(l:C) used were endotoxin-free. Mice were divided into four groups treated with Sham as control, Poly(l:C), mechanical ventilation with tidal volume of 10mL/kg (MTV) and Poly(l:C) followed with MTV as indicated in the figure. Results are shown as means \pm SEM $(n=6)$ and compared by one-way ANOVA and Student-Newman-Keuls test. ${ }^{\star \star} p<0.01,{ }^{\star \star \star} p<0.001$

alone (Figure 3D). These findings indicate that caspase-1 contributes to IL-6, IL-1 $\beta$ and IL-18 release into the circulation.

\section{Regulation of Caspase-11 Expression and Activation, Its Relationship to Caspase-1 Pathways in Whole Lung and Isolated Macrophages After Poly(I:C), MTV and Their Combination in Intact Mice}

We quantified expression and activation of caspase-11 and caspase-1 in whole lungs and freshly isolated alveolar macrophages by Western blot after Poly(I:C) and/or MTV. Compared to the control group, there was a slight increase of procaspase-11 after Poly(I:C) administration while procaspase11 expression was not affected by MTV alone. MTV after Poly (I:C) priming resulted in a significant increase in procaspase-11 in whole lung (Figure $\mathbf{4 A}$ ) and alveolar macrophages (Figure 4B). MTV after Poly(I:C) also resulted in a large increase in the appearance of cleaved caspase-11 in whole lung (Figure 4A) and alveolar macrophages (Figure 4B). Overall levels of procaspase-1 were not influenced by either stimuli alone (data not shown). There was a slight increase in cleaved caspase-1 after Poly(I:C) but not MTV and this was greatly increased in the combined protocol in both whole lung (Figure 4A) and alveolar macrophages (Figure 4B). Since caspase-11 may affect caspase-1 activity (30), we repeated these experiments in alveolar macrophages from caspase-11-/- mice and noted that intrapulmonary cleaved caspase-1 levels were significantly lower in both Poly(I:C) and Poly(I:C)+MTV in caspase-11-/- mice compared to WT mice (Figure 4C). Nonetheless, cleaved caspase- 1 was still induced in caspase11-/- mice underscoring the partial interdependence of caspases- 1 and -11 .

We assessed the activation of caspase-11 in advanced glycosylation end product-specific receptor (RAGE) knock-out mice. Supplementary Data 1 showed that the pro-caspase-11 expression was decreased by block of RAGE after Poly(I:C)+ MTV, and activation of caspase- 11 was significantly inhibited in RAGE-/- mice, indicating that gut-lung axis pathway may depended on RAGE.

NLRP3 is part of a common canonical inflammasome that includes apoptosis-associated speck-like protein (ASC), caspase activation and recruitment domain (CARD) and caspase-1 (21). Figure 5A shows a significant increase in NLRP3 mRNA level in alveolar macrophages in Poly(I:C) group compared to sham 
A

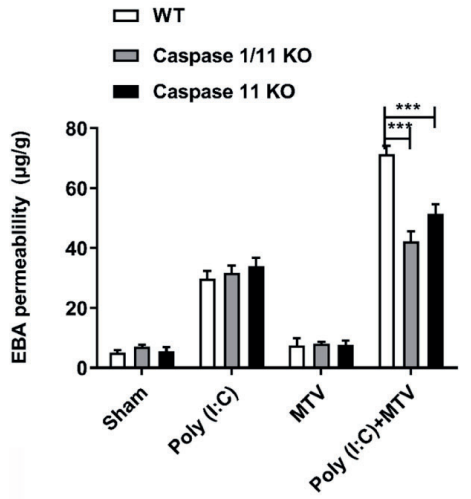

C

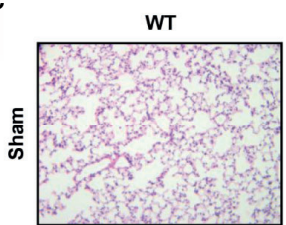

Caspase-1/11 KO
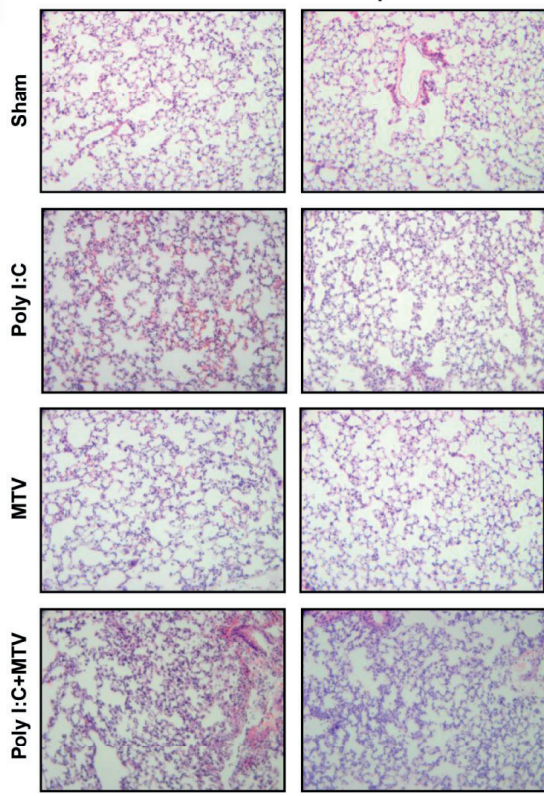

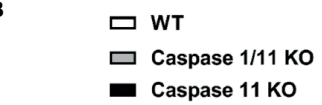

IIIn

Caspase-11 KO
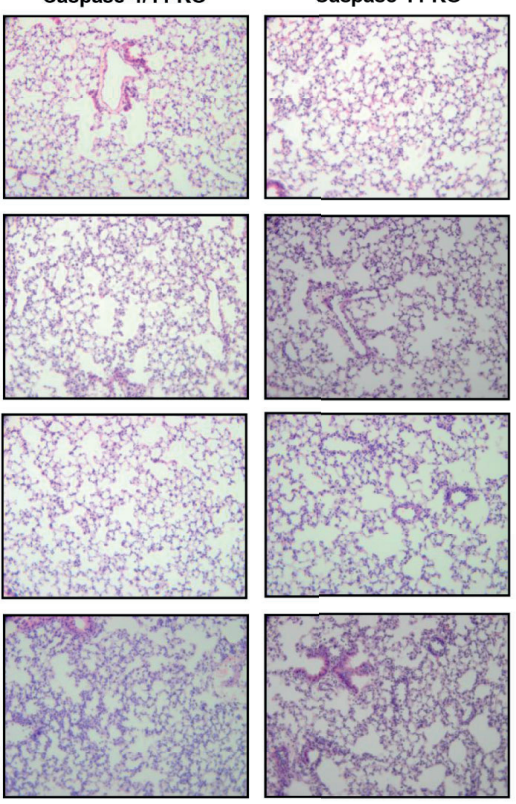

D

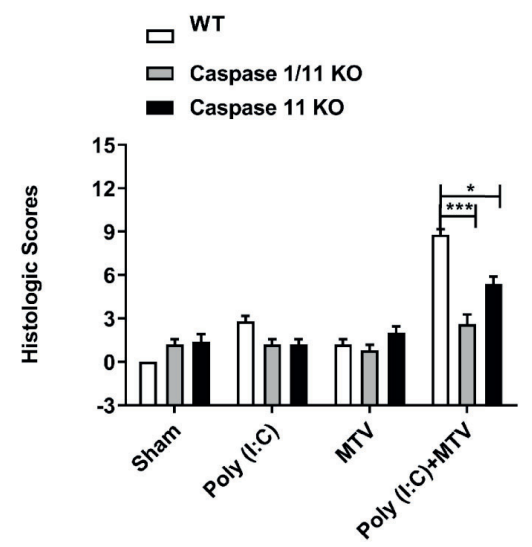

FIGURE 2 | Caspase-1 and caspase-11 prevented Poly(l:C)-MTV induced lung injury. Wildtype (WT), Caspase-1/11 null and Caspase-11 null mice (Caspase 1/11 KO and Caspase $11 \mathrm{KO}$ ) were treated with four groups as indicated. EBA permeability $(\mathrm{n}=5) \mathbf{( A )}$, total protein concentration in BALF $(\mathrm{n}=6$ for $\mathrm{WT}$ mice, $\mathrm{n}=12$ for caspase$1 / 11$ and caspase-11 KO mice) (B), H\&E histology $(n=5)(\mathbf{C})$ of the lung cross section from WT, caspase-1/11 KO, caspase-11 KO mice (Scale bars: 50 um) and total histopathologic scores of lung injury (D) were evaluated by two different authors calculated for each animal. Results are shown as means \pm SEM and compared by one-way ANOVA and Student-Newman-Keuls test. ${ }^{*} p<0.05,{ }^{\star \star \star} p<0.001$. 
A

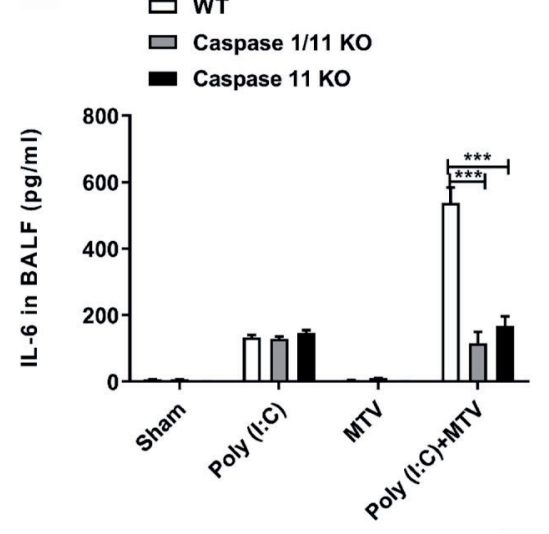

c

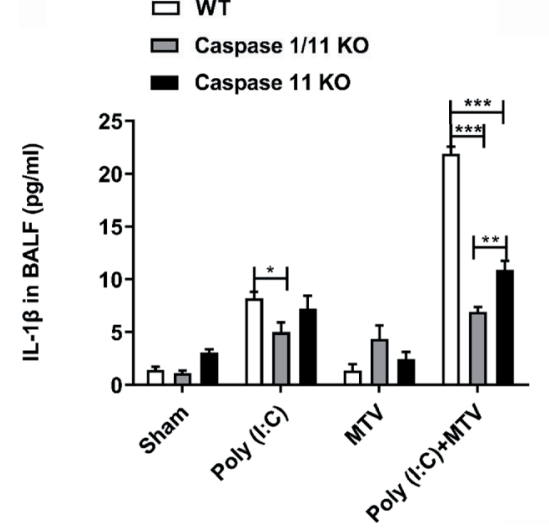

B

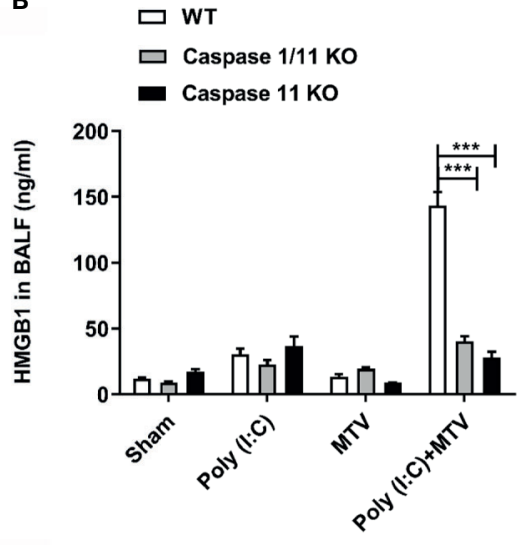

D $\square \mathrm{WT}$

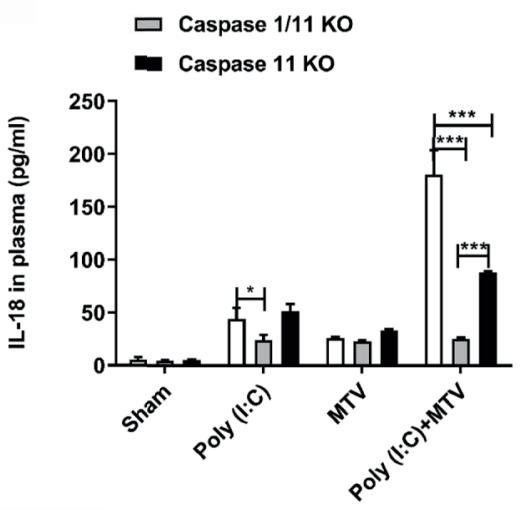

FIGURE 3 | Caspase-1 and caspase-11 alleviated Poly(l:C)-MTV induced pulmonary informatory response. IL-6 (A), HMGB1 ( $\mathrm{n=5}$ ) (B) and IL-1 3 (C) in BALF as well as IL-18 levels (D) in plasma were significantly decreased in Caspase 1/11 KO and Caspase-11 KO mice compared to WT mice. In (A, C, D), n=6 for WT mice, $\mathrm{n}=12$ for caspase-1/11 and caspase-11 KO mice. Results are shown as means \pm SEM and compared by one-way ANOVA and Student-Newman-Keuls test. ${ }^{\star \star} p<0.01,{ }^{\star \star \star} p<0.001$.

group. There was no difference between the sham and MTV groups. The combination of Poly(I:C) and MTV greatly increased NLRP3 mRNA and this was partially sensitive to genetic ablation of caspase-11 potentially placing caspase-11 upstream of this canonical inflammasome. The role of NLRP3 was further assessed by measuring IL-6 (Figure 5B) and IL-1 $\beta$ (Figure 5C) secretion in BALF and IL-18 (Figure 5D) release in plasma of wildtype and NLRP3-/- mice. As expected, increases in these cytokines after Poly(I:C) and combined Poly(I:C)-MTV were sensitive to ablation of NLRP3. These data underscore the interdependent role of caspase-11 and caspase-1 in combined Poly(I:C)-MTV acute lung injury.

\section{Caspase-11 Cleavage of GSDMD and Poly (I:C)-MTV ALI Including Pyroptosis in Freshly Isolated Alveolar Macrophages From Injured Lung}

Activation of inflammatory caspases-11 and -1 may lead to cleavage mediated activation of GSDMD that in turn is an obligatory step in inflammatory programmed cell death (pyroptosis). We quantified expression and regulation of GSDMD in alveolar macrophage by Western blot after Poly(I: C) and/or MTV (Figure 6). MTV after Poly(I:C) resulted in a significant increase in appearance of cleaved GSDMD in alveolar macrophages (Figure 6).

Accordingly, we repeated experiments with Poly(I:C), MTV and their combination in GSDMD-/- mice and noted GSDMD sensitive Poly(I:C)-MTV mediated lung injury [as determined by evans blue permeability (Figure $7 \mathbf{A}$ ) and protein in BALF (Figure 7B)] and increased cytokine release (Figures 7C-F).

GSDMD sensitive injury is consistent with pyroptosis and thus we determined whether pyroptosis occurred in freshly isolated alveolar macrophages from wildtype mice after Poly (I:C), MTV or their combination (Figure 8). Macrophages, neutrophils and lymphocytes in BALF were harvested to assess the cell recruitments by $\operatorname{Poly}(\mathrm{I}: \mathrm{C}) / \mathrm{MTV}$. Here we show a significant increase of total cell numbers in BALF in Poly(I:C) + MTV group compared that in sham, Poly(I:C) or MTV group, and the neutrophils contributed the most increase (78\%) in the BALF (Supplementary Data 2). 

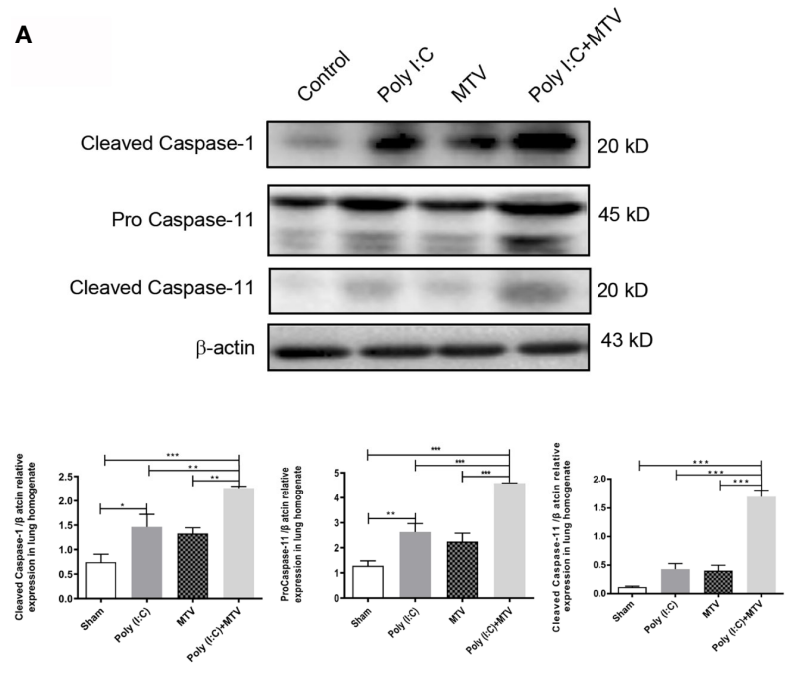

B
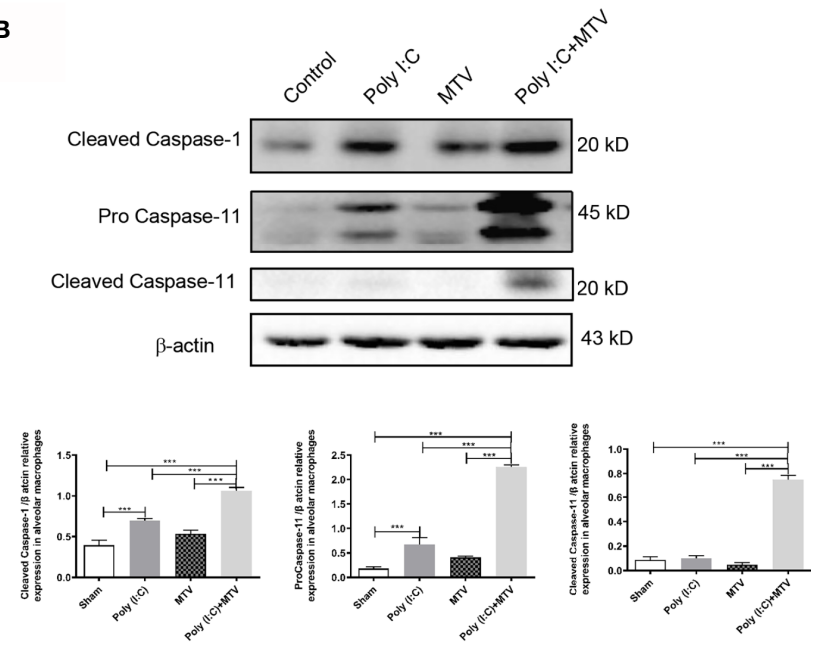

C
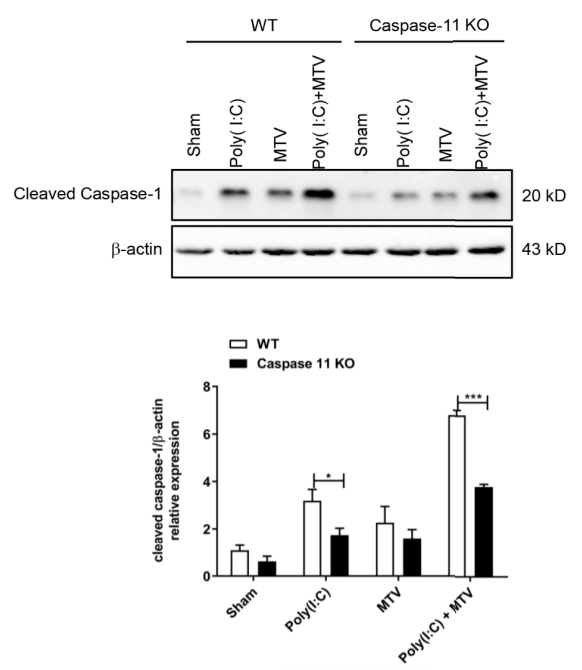

FIGURE 4 | Continued
FIGURE 4 | Alterations of protein levels of cleaved caspase-1, procaspase-11 and cleaved caspase-11 following Poly(l:C)-MTV. MTV following Poly(l:C) instillation resulted in a significant increase in procapase-11 and, large increase in appearance of cleaved caspase-11 in whole lung (A) and alveolar macrophages (B). Cleaved caspase-1 expression levels were inhibited in caspase-11 KO mice in Poly (I:C)-MTV compared to that in WT mice (C). Results are shown as means \pm SEM $(n=12)$ and compared by one-way ANOVA and Student-Newman-Keuls test. ${ }^{*} p<0.05,{ }^{* *} p<0.01,{ }^{* \star} p<0.001$.

Alveolar macrophages were harvested at end of exposure and stained with Alexa Fluor 488-labeled caspase-1 FLICA, Alexa Fluor 546-labeled in situ cell death reagent-TMR and Hoechst dye (Figure 8A). Quantitation of colocalization of caspase-1 and TUNEL positive cells showed slight increase in pyroptosis after either Poly(I:C) or MTV and a very large increase after combined exposure in situ (Figure 8B).

\section{DISCUSSION}

We note that otherwise non-injurious moderate tidal volume ventilation exacerbates ALI after i.t. Poly(I:C) in intact mice. Disruption of the alveolar-capillary barrier (Figures $\mathbf{1}$ and 2) in this two-hit model, as previously reported by Chun et al. (21) and us (20), is a central feature of ALI and thus provides a useful preclinical framework in identifying pathways that underscore the major contribution of sepsis to ARDS. Although epidemiologic studies suggest that direct (e.g. pneumonia) sepsis comprises a large component of the risk factors in the development of ARDS, a less understood multifactorial extrapulmonary (indirect or systemic) sepsis is also important (2). In the current study, we note (Figure 1) that combined Poly (I:C)-MTV enhanced permeability of gastrointestinal tract and introduced endotoxin to the vascular space and lung. The association of systemic sepsis and ALI (via gut-lung axis) was reinforced by intracellular activation of caspase-11 in the lungs (Figure 4A) and alveolar macrophages (Figure 4B). Systemic endotoxemia also activated caspase-1 and non-canonical activation of inflammasome (Figure 5) with synthesis and release (Figures 3, 5 and 6) of IL-1 cytokines (IL-1 $\beta$ and IL18) and HMGB1. Interactions between caspase-11 and caspase- 1 led to GSDMD dependent barrier disruption (Figure 7) and pyroptosis in alveolar macrophages (Figure 8). A schema outlining these pathways is presented in Figure $\mathbf{9}$ and underscores the complexities of direct lung injury combined with extrapulmonary sepsis.

Mechanical ventilation is a common clinical strategy to rest injured lung and improve gas exchange in critical care setting (3) and to deliver anesthetic agents intraoperatively. The proinflammatory and injurious nature of high tidal volume ventilation has led to adherence to lung-protective ventilation protocols minimizing ventilator induced lung injury (VILI) and greatly reducing morbidity and mortality in ARDS (2-4). Nonetheless, otherwise non-injurious lower tidal volume ventilation may exacerbate preexisting acute lung injury due to bacterial $(5,6)$ or viral $(11)$ infection, intratracheal endotoxin 

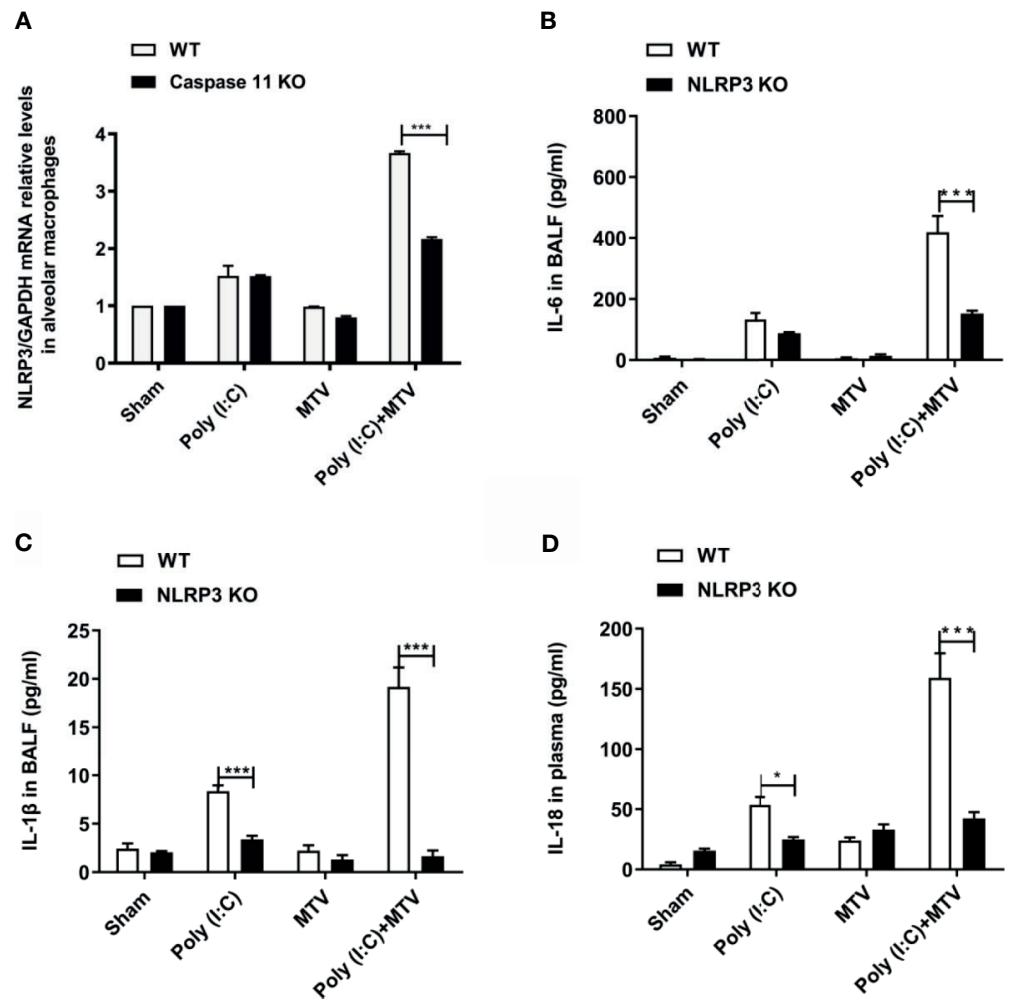

FIGURE 5 | NLRP3 was required for Poly(l:C)-MTV induced lung injury. Poly(l:C)-MTV increased NLRP3 mRNA levels in alveolar macrophages in WT mice but was partially inhibited by caspase-11 KO mice, ( $n=12)$ (A). NLRP3 was required for Poly(I:C)-MTV induced IL-6 (B), IL-1 3 release in BALF (C) and IL-18 (D) secretion in plasma. In (B-D), $n=6$ of each group for WT mice, $n=8$ for $\mathrm{NLRP3}^{-/}$mice. Results are shown as means \pm SEM and compared by one-way ANOVA and StudentNewman-Keuls test. ${ }^{\star \star \star} p<0.001$.

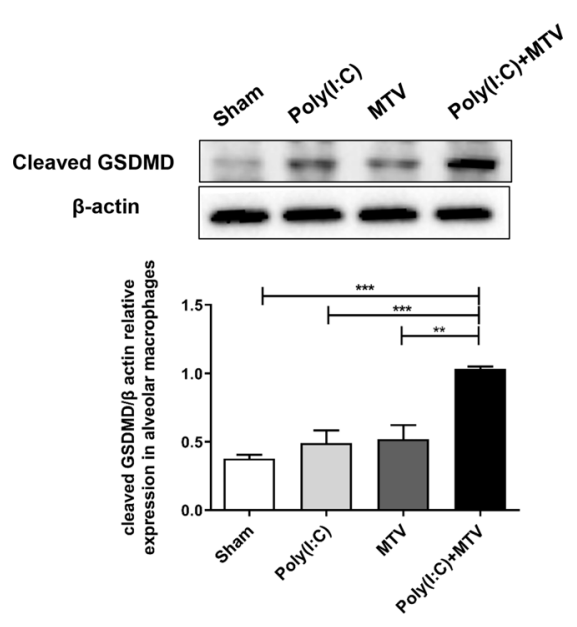

FIGURE 6 | Alterations of protein levels of cleaved GSDMD in alveolar macrophages following Poly(I:C)-MTV. Compared to the sham group, there was no significant increase in GSDMD expression in alveolar macrophages from mice treated with Poly(l:C) or MTV alone. MTV following Poly(l:C) instillation resulted in a significant increase of cleaved GSDMD in alveolar macrophages. Results are shown as means \pm SEM $(n=4)$ and compared by one-way ANOVA and Student-Newman-Keuls test. ${ }^{\star \star} p<0.01,{ }^{\star \star \star} p<0.001$.
(7-10) or sterile injury such as hyperoxia (14) or acid instillation $(12,13)$. Sensitization of VILI to events originating at distal sites or plasma space including exogenous endotoxin $(16,17)$ or polymicrobial sepsis $(18,19)$ has also been documented. We confirmed (20) that MTV exacerbated modest acute lung injury secondary to intratracheal instillation of Poly(I:C). Poly(I:C), a TLR3 ligand, is a synthetic analog of double stranded RNA that can be produced by many viruses during their replicative cycles (31). The precise mediators or pathways underlying the synergistic effect of Poly(I:C) and MTV are unknown but are largely independent of TLR4 (21). In the current study, we add the possibility that extrapulmonary endotoxemia secondary to enhanced gastrointestinal permeability with Poly(I:C)-MTV underlies this synergistic effect and reveal a role for intracellular LPS mediated caspase-11 activation, a noncanonical inflammasome pathway and interactions of caspase11 and caspase- 1 in lung injury.

The influence of interactions between gut and lung microbiota in respiratory health is firmly established in chronic (32) and acute $(23,24)$ disease. Evidence of such microbial mingling in ARDS has suggested therapeutic strategies for ARDS of probiotics (33), novel bio-engineered delivery systems (34) and antimicrobial agents (23). In the current study, combined injury led to an increase in gut permeability with the 

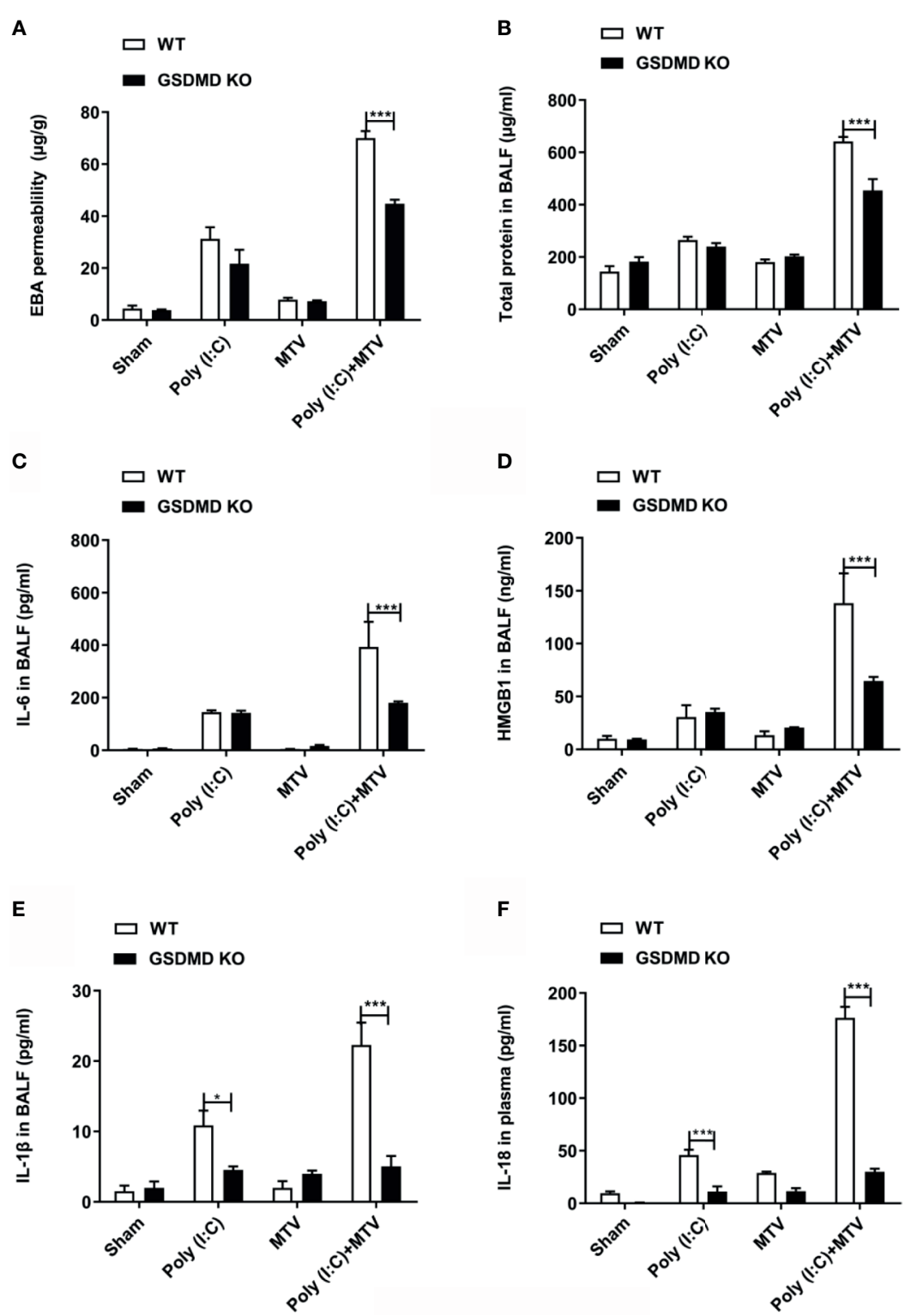

FIGURE 7 | GSDMD was required for Poly(l:C)-MTV induced lung injury. Poly(l:C)-MTV increased EBA permeability, (A), total protein (B), IL-6 (C), HMGB1 (D), and $\mathrm{IL}-1 \beta \mathbf{~ ( E )}$ in BALF as well as IL-18 levels (F) in plasma in WT but were inhibited in GSDMD KO mice. $n=5$ of each group for WT mice and GSDMD ${ }^{-1-}$ mice. Results are shown as means \pm SEM and compared by one-way ANOVA and Student-Newman-Keuls test. ${ }^{\star \star \star} p<0.001$.

introduction of endotoxin to the circulation and lung (Figure 1). We did not attempt to identify mediators released from lung that might account for this effect on the gut but it is noteworthy that macrophages isolated from Poly(I:C)-MTV treated mice (20) release tumor necrosis factor (TNF)- $\alpha$ and anti-TNF- $\alpha$ antibodies have been shown to abrogate the increase in gut permeability (and lung edema) in high volume ventilation in rats (35). We assumed that endotoxemia in our model was secondary to increased gut permeability as Poly(I:C) mixture was endotoxin free and neither intubation nor circuitry for mechanical ventilation introduced significant amounts of endotoxin in lung (Figure 1D). The identification of systemic endotoxemia in the combined Poly(I:C)-MTV protocol motivated us to pursue caspase-11 mediated pyroptosis in lung as Chun et al. (21) reported a TLR-4 independent pathway in this model and Cheng et al. (25) noted that introduction of systemic endotoxin caused TLR-4 independent, caspase-11 mediated pyroptosis in mice. HMGB1 is known to deliver extracellular LPS via RAGE to cytosolic caspase-11 (36). We observed elevated levels of HMGB1 after Poly(I:C)-MTV and implicate RAGE in the pulmonary changes after Poly(I:C)-MTV by showing that RAGE-/- mice fail to increase procaspase-11 and 

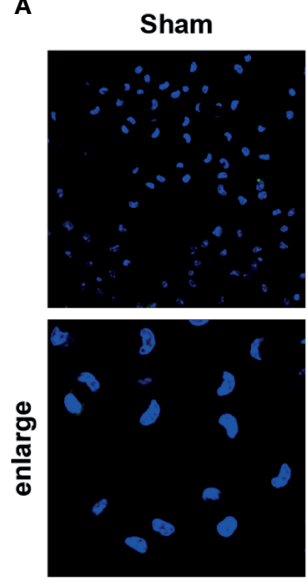

西
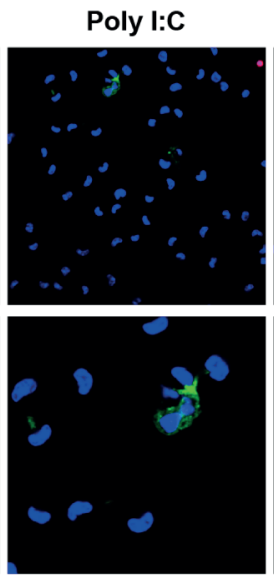

active caspase-1 TUNEL Hoechst

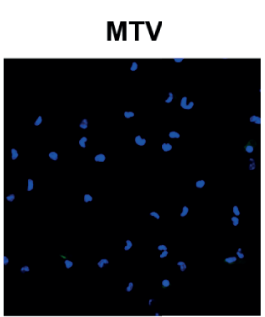

\section{Poly I:C+MTV}
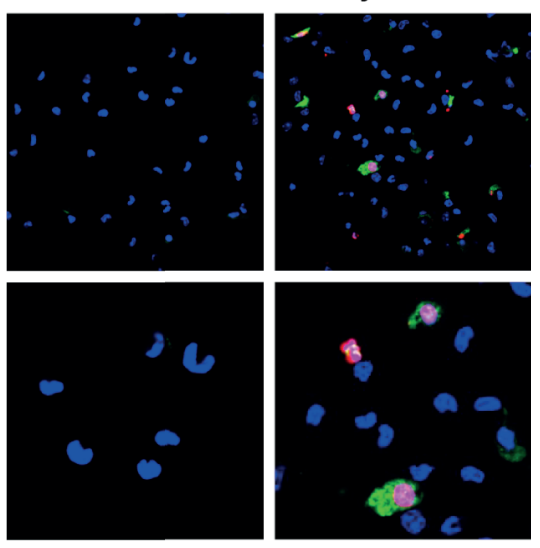

B

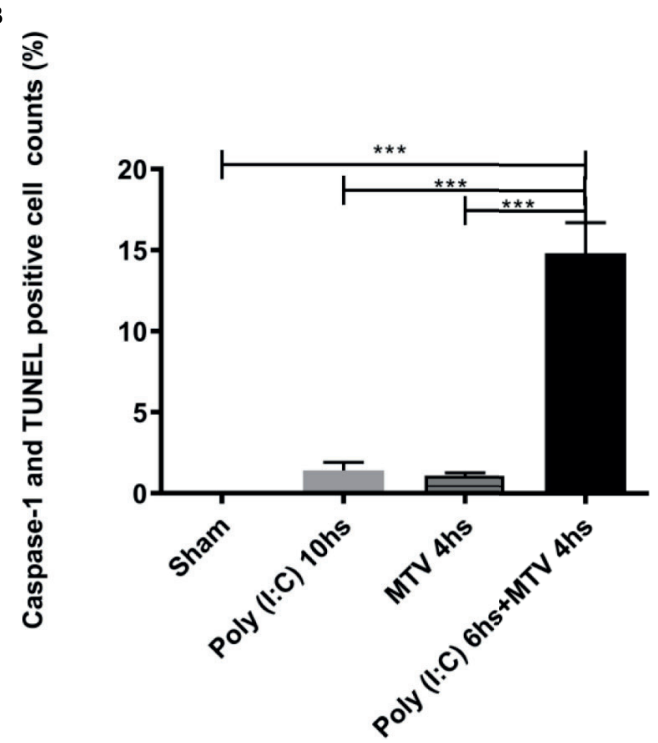

FIGURE 8 | MTV induced alveolar macrophage pyroptosis after Poly(l:C) priming. Alveolar macrophages on and maturation and release of cytokines in combined Poly(l:C)-MTV injury model. The gut-lung axis resulted in activation of pulmonary non-canonical (cytosolic mediated caspase-11 activation) and canonical (Caspase-1) inflammasome (NLRP3) mediated ALI in this two hit model resulting in GSDMD sensitive alveolar capillary barrier disruption, pyroptosis (in alveolar macrophages) and cytokine maturation and release (IL-1 $\beta$; IL-18) were isolated immediately after mice scarification and adhere for at least 2 hours before staining. Caspase-1 activation was labeled with FLICA caspase-1 and DNA fragmentation was labeled with TUNEL by confocal microscopy (A). Quantification was done by Image $\mathrm{J}$ (B). Results are shown as means \pm SEM $(n=12)$ and compared by one-way ANOVA and Student-Newman-Keuls test. ${ }^{* \star *} p<0.001$.

cleaved caspase-11 in lungs of mice subjected to Poly(I:C)-MTV (Supplementary Data 1). Future experiments to neutralize systemic endotoxemia or eliminate of gut microbes in general (e.g. gnobiotic mice or combined antibiotic therapies) will help advance gut-lung axis hypothesis beyond associative observations in the current study.

Pyroptosis is an inflammatory programmed cell death pathway activated by murine caspase- 1 or caspase-11 (caspase4 and -5 are human orthologs) and requires cleavage and activation of pore-forming effector protein, GSDMD (27). It appears to be a key component of innate immunity and teleologically is an effective means of eliminating intracellular pathogens and signaling host via release of inflammatory mediators (25). Nonetheless, excessive activation is implicated in human diseases including sepsis (37). For example, dihydromyricetin, an inhibitor of NLRP3, alleviated cecal ligation and puncture-induced lung histopathologic injury in mice (38). As cell death, per se, and inflammatory mediators are essential components of disruption of alveolar capillary barrier in ALI and ARDS, insight into relevant pathways may be informative of pathogenesis and therapeutic strategies. Caspase-1 activation is well known to be activated via a canonical inflammasome pathway (including but not limited to NLRP3) as well as a caspase-11 mediated non-canonical 


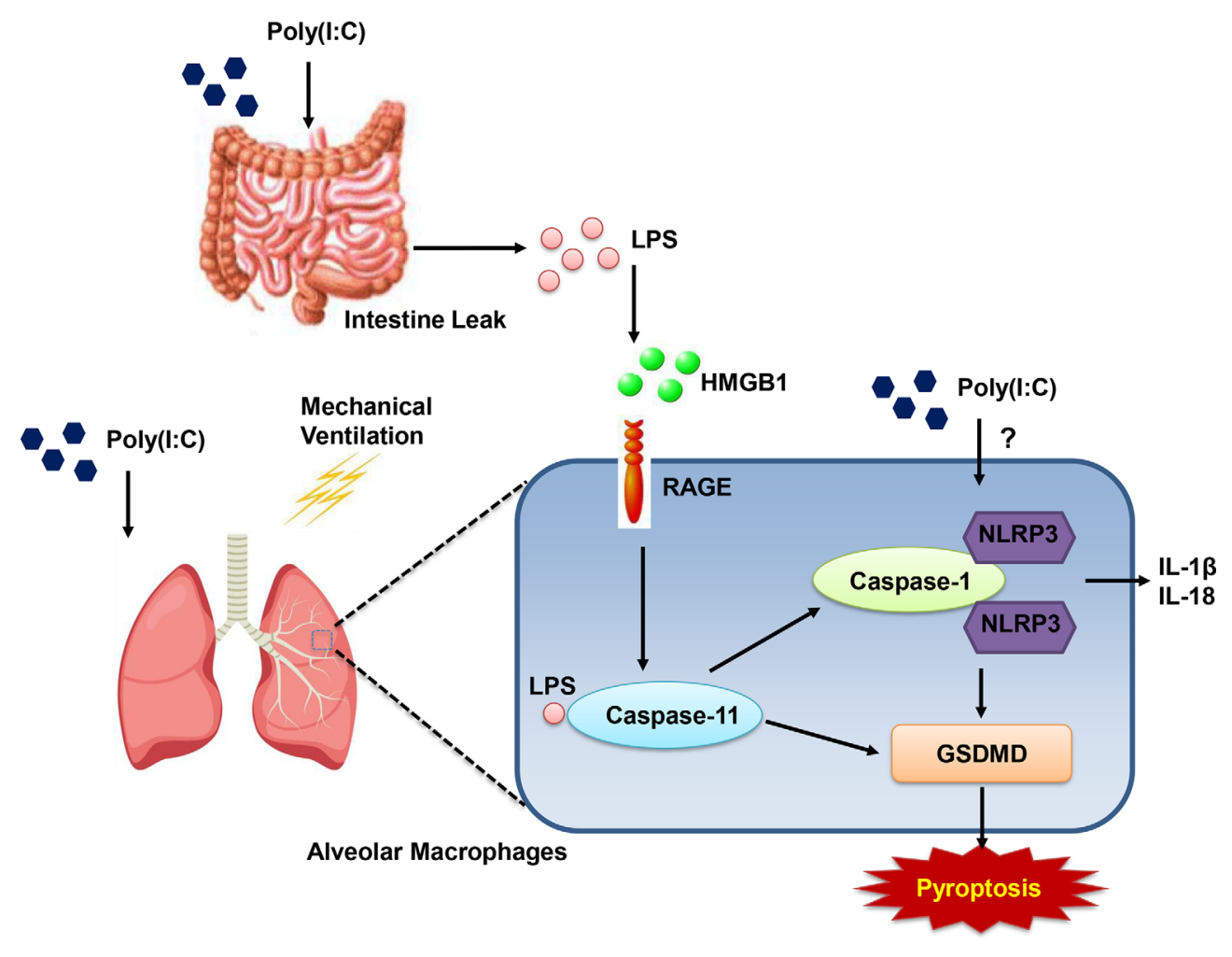

FIGURE 9 | Schematic graph of gut-lung axis. Combined (Poly(l:C)-MTV) insult results in increase in gastrointestinal permeability and endotoxin in plasma and BALF. Poly(l:C)+MTV insult was sensitive to Caspase-11 deletion with no further contribution of caspase-1 but led to large increases in procaspase 11 and its cleaved product as well as cleaved product of caspase-1. Genetic ablation of Gasdermin D (GSDMD) attenuated alveolar-capillary disruption.

inflammasome pathway (27). Canonical activators include dsRNA [and mechanical ventilation (39)] as well as bacteria; non-canonical activators include gram negative bacteria. Accordingly, there is interaction of these caspases in the maturation and release of cytokines of IL-1 family (IL-1 $\beta$ and IL-18), as well as pyroptosis $(27,30)$. In the current study, Poly(I: C) is likely to activate the canonical pathway as noted by: a) increase in mRNA of NLRP3 (Figure 5A) in alveolar macrophages of Poly(I:C)-MTV treated mice that was only partially sensitive in the caspase-11 null mice; and b) synthesis and release of IL-1 $\beta$, IL- 6 and IL-18 that was sensitive to genetic deletion of NLRP3 (Figures 5B-D). Mechanical ventilation has also been shown (39) to activate NLRP3 inflammasome in alveolar macrophages in a caspase-1 dependent fashion underscoring an additional stimulus of canonical pathway in our combined Poly(I:C)-MTV model. Alveolar-capillary barrier disruption was only partially sensitive to genetic ablation of GSDMD (Figures 7A, B) as was release of alarmins (HMGB1; Figure 7D) whereas release of IL-18 to plasma space (Figure 7F) was highly sensitive to GSDMD deletion in combined injury protocol further underscoring the interaction of these pathways and resultant phenotype (Figure 9). To the best of our knowledge, in situ identification of cellular components of pyroptosis remains challenging in murine tissue. As such, we utilized an ex vivo strategy involving isolation and short-term culture of murine alveolar macrophages from Poly(I:C)-MTV treated mice (Figure 8) and quantified pyroptosis via coexpression of caspase-1 and TUNEL. Although macrophages (and precursor monocytes) are prototypic of death by pyroptosis, it is noteworthy that other investigators have utilized primary cultures of murine pulmonary endothelial cells isolated from systemic endotoxemic mice (25) or cultured pulmonary epithelial cells (35) directly exposed, in vitro, to reveal presence (and differences) in pyroptosis and release of cytokines. Within the limits of our study, we suggest that combined Poly(I:C)-MTV activates both canonical and non-canonical inflammasome pathways involving both caspase-11, caspase-1 and their interaction and GSDMD dependent pyropotosis in at least alveolar macrophages (Figure 9). Further cellular origins, aside from alveolar macrophages, awaits improvements in antibody dependent immunohistochemistry in murine lung and pharmacologic separation of caspase-1 and caspase-11 and relevant inflammasome pathways.

In conclusion, By revisiting (25) a two hit model (Poly(I:C)MTV) of acute lung injury noted to be TLR4 independent, we have detected an additional stimulus, e.g. systemic endotoxemia; as a result of gut-lung axis, both non-canonical caspase-11 [via presumptive cytosolic endotoxemia (28)] and canonical [via 
NLRP3 inflammasome $(38,39)]$ and their interactions led to pyroptosis in alveolar macrophages, disruption of alveolar capillary barrier and proinflammatory state within lung. Pharmacologic strategies at disrupting communication between gut and lung, inhibition of inflammasomes or effector molecules (GSDMD) in pyroptosis may be useful in acute lung injury.

\section{DATA AVAILABILITY STATEMENT}

The datasets presented in this study can be found in online repositories. The names of the repository/repositories and accession number(s) can be found in the article/Supplementary Material.

\section{ETHICS STATEMENT}

The animal study was reviewed and approved by Animal Care and Use Committee.

\section{AUTHOR CONTRIBUTIONS}

SJ, XD, TB, QL, and L-MZ have made contributions to research concept and design.SJ, XD and MD have made contributions to the acquisition, analysis of data. SJ, HL, BP,TB, and L-MZ have made contributions to data interpretation. CY and WL have made contributions to the creation of new software used in the work. SJ, $\mathrm{XL}, \mathrm{BP}, \mathrm{TB}$, and L-MZ have made manuscript preparation. SJ, BP, $\mathrm{TB}, \mathrm{QL}$, and L-MZ have critically revised of the manuscript and approved manuscript final version. All authors contributed to the article and approved the submitted version.

\section{REFERENCES}

1. Bellani G, Laffey JG, Pham T, Fan E, Brochard L, Esteban A, et al. Epidemiology, Patterns of Care, and Mortality for Patients With Acute Respiratory Distress Syndrome in Intensive Care Units in 50 Countries. JAMA (2016) 315(8):788-800. doi: 10.1001/jama.2016.0291

2. Englert JA, Bobba C, Baron RM. Integrating Molecular Pathogenesis and Clinical Translation in Sepsis-Induced Acute Respiratory Distress Syndrome. JCI Insight (2019) 4(2):e124061. doi: 10.1172/jci.insight.124061

3. Slutsky AS, Ranieri VM. Ventilator-Induced Lung Injury. N Engl J Med (2013) 369(22):2126-36. doi: 10.1056/NEJMra1208707

4. Matthay MA, Ware LB, Zimmerman GA. The Acute Respiratory Distress Syndrome. J Clin Invest (2012) 122(8):2731-40. doi: 10.1172/JCI60331

5. Dhanireddy S, Altemeier WA, Matute-Bello G, O’Mahony DS, Glenny RW, Martin TR, et al. Mechanical Ventilation Induces Inflammation, Lung Injury, and Extra-Pulmonary Organ Dysfunction in Experimental Pneumonia. Lab Invest (2006) 86(8):790-9. doi: 10.1038/labinvest.3700440

6. Muller-Redetzky HC, Will D, Hellwig K, Kummer W, Tschernig T, Pfeil U, et al. Mechanical Ventilation Drives Pneumococcal Pneumonia Into Lung Injury and Sepsis in Mice: Protection by Adrenomedullin. Crit Care (2014) 18 (2):R73. doi: 10.1186/cc13830

7. Altemeier WA, Matute-Bello G, Gharib SA, Glenny RW, Martin TR, Liles WC. Modulation of Lipopolysaccharide-Induced Gene Transcription and

\section{FUNDING}

This work was supported in part by the National Natural Science Foundation of China (No. 81772114 to QL, No.81971882 to QL) and Development fund of anesthesiology, Shanghai Pulmonary Hospital (XL) for the design of the study, collection and writing the manuscript, analysis and interpretation of data. Salaries for SJ, XD, CY, WL, XL, and QL were supported by their original hospitals.

\section{ACKNOWLEDGMENTS}

The authors thank Ms. Debra Williams for technical and logistical support and assistance.

\section{SUPPLEMENTARY MATERIAL}

The Supplementary Material for this article can be found online at: https://www.frontiersin.org/articles/10.3389/fimmu.2021. 693874/full\#supplementary-material

Supplementary Data 1 | Alterations of protein levels of procaspase-11 and cleaved caspase-11 are dependent on RAGE in the Poly(l:C)-MTV model.MTV following Poly(l:C) instillation resulted in a significant increase in procapase-11 and cleaved caspase-11 in alveolar macrophages from WT mice. The increases were not observed in RAGE KO mice in Poly (I:C)-MTV compared to that in WT mice. Results are shown as means \pm SEM $(n=4)$ and compared by one-way ANOVA and Student-Newman-Keuls test. ${ }^{* \star} p<0.001$.

Supplementary Data 2 | Cell recruitment and classified cell counts in BALF after Poly(l:C)-MTV.MTV following Poly(l:C) instillation resulted in a significant increase of total cell numbers in BALF (A). Neutrophils increased most among all the cells (B). Results are shown as means \pm SEM $(n=4)$ and compared by one-way ANOVA. ${ }^{\star \star \star} p<0.001$.

Promotion of Lung Injury by Mechanical Ventilation. J Immunol (2005) 175 (5):3369-76. doi: 10.4049/jimmunol.175.5.3369

8. Hu G, Malik AB, Minshall RD. Toll-Like Receptor 4 Mediates Neutrophil Sequestration and Lung Injury Induced by Endotoxin and Hyperinflation. Crit Care Med (2010) 38(1):194-201. doi: 10.1097/CCM.0b013e3181bc7c17

9. Ding N, Wang F, Xiao H, Xu L, She S. Mechanical Ventilation Enhances HMGB1 Expression in an LPS-Induced Lung Injury Model. PLoS One (2013) 8(9):e74633. doi: 10.1371/journal.pone.0074633

10. Rentsendorj O, Damarla M, Aggarwal NR, Choi JY, Johnston L, D’Alessio FR, et al. Knockdown of Lung Phosphodiesterase 2A Attenuates Alveolar Inflammation and Protein Leak in a Two-Hit Mouse Model of Acute Lung Injury. Am J Physiol Lung Cell Mol Physiol (2011) 301(2):L161-70. doi: 10.1152/ajplung.00073.2011

11. Bem RA, van Woensel JB, Bos AP, Koski A, Farnand AW, Domachowske JB, et al. Mechanical Ventilation Enhances Lung Inflammation and Caspase Activity in a Model of Mouse Pneumovirus Infection. Am J Physiol Lung Cell Mol Physiol (2009) 296(1):L46-56. doi: 10.1152/ajplung.00467.2007

12. Frank JA, Gutierrez JA, Jones KD, Allen L, Dobbs L, Matthay MA. Low Tidal Volume Reduces Epithelial and Endothelial Injury in Acid-Injured Rat Lungs. Am J Respir Crit Care Med (2002) 165(2):242-9. doi: 10.1164/ajrccm.165.2.2108087

13. Kuiper JW, Plotz FB, Groeneveld AJ, Haitsma JJ, Jothy S, Vaschetto R, et al. High Tidal Volume Mechanical Ventilation-Induced Lung Injury in Rats Is Greater After Acid Instillation Than After Sepsis-Induced Acute Lung Injury, 
But Does Not Increase Systemic Inflammation: An Experimental Study. BMC Anesthesiol (2011) 11:26. doi: 10.1186/1471-2253-11-26

14. Makena PS, Luellen CL, Balazs L, Ghosh MC, Parthasarathi K, Waters CM, et al. Preexposure to Hyperoxia Causes Increased Lung Injury and Epithelial Apoptosis in Mice Ventilated With High Tidal Volumes. Am J Physiol Lung Cell Mol Physiol (2010) 299(5):L711-9. doi: 10.1152/ajplung.00072.2010

15. Yehya N, Xin Y, Oquendo Y, Cereda M, Rizi RR, Margulies SS. Cecal Ligation and Puncture Accelerates Development of Ventilator-Induced Lung Injury. Am J Physiol Lung Cell Mol Physiol (2015) 308(5):L443-51. doi: 10.1152/ ajplung.00312.2014

16. Altemeier WA, Matute-Bello G, Frevert CW, Kawata Y, Kajikawa O, Martin TR, et al. Mechanical Ventilation With Moderate Tidal Volumes Synergistically Increases Lung Cytokine Response to Systemic Endotoxin. Am J Physiol Lung Cell Mol Physiol (2004) 287(3):L533-42. doi: 10.1152/ ajplung.00004.2004

17. Bregeon F, Delpierre S, Chetaille B, Kajikawa O, Martin TR, Autillo-Touati A, et al. Mechanical Ventilation Affects Lung Function and Cytokine Production in an Experimental Model of Endotoxemia. Anesthesiology (2005) 102(2):3319. doi: 10.1097/00000542-200502000-00015

18. Ding X, Tong Y, Jin S, Chen Z, Li T, Billiar TR, et al. Mechanical Ventilation Enhances Extrapulmonary Sepsis-Induced Lung Injury: Role of WISP1Alphavbeta5 Integrin Pathway in TLR4-Mediated Inflammation and Injury. Crit Care (2018) 22(1):302. doi: 10.1186/s13054-018-2237-0

19. Ding X, Jin S, Shao Z, Xu L, Yu Z, Tong Y, et al. The IL-33-ST2 Pathway Contributes to Ventilator-Induced Lung Injury in Septic Mice in a Tidal Volume-Dependent Manner. Shock (2019) 52(3):e1-e11. doi: 10.1097/ SHK.0000000000001260

20. Jin S, Chen Z, Ding X, Zhao X, Jiang X, Tong Y, et al. Mechanical Ventilation Augments Poly(I:C)induced Lung Injury via a WISP1-Integrin Beta3 Dependent Pathway in Mice. Mol Med (2016) 22:54-63. doi: 10.2119/ molmed.2015.00233

21. Chun CD, Liles WC, Frevert CW, Glenny RW, Altemeier WA. Mechanical Ventilation Modulates Toll-Like Receptor-3-Induced Lung Inflammation via a MyD88-Dependent, TLR4-Independent Pathway: A Controlled Animal Study. BMC Pulm Med (2010) 10:57. doi: 10.1186/1471-2466-10-57

22. Mittal R, Coopersmith CM. Redefining the Gut as the Motor of Critical Illness. Trends Mol Med (2014) 20(4):214-23. doi: 10.1016/j.molmed.2013.08.004

23. Dickson RP, Singer BH, Newstead MW, Falkowski NR, Erb-Downward JR, Standiford TJ, et al. Enrichment of the Lung Microbiome With Gut Bacteria in Sepsis and the Acute Respiratory Distress Syndrome. Nat Microbiol (2016) 1 (10):16113. doi: 10.1038/nmicrobiol.2016.113

24. Mukherjee S, Hanidziar D. More of the Gut in the Lung: How Two Microbiomes Meet in ARDS. Yale J Biol Med (2018) 91(2):143-9.

25. Cheng KT, Xiong S, Ye Z, Hong Z, Di A, Tsang KM, et al. Caspase-11Mediated Endothelial Pyroptosis Underlies Endotoxemia-Induced Lung Injury. J Clin Invest (2017) 127(11):4124-35. doi: 10.1172/JCI94495

26. Kayagaki N, Stowe IB, Lee BL, O’Rourke K, Anderson K, Warming S, et al. Caspase-11 Cleaves Gasdermin D for Non-Canonical Inflammasome Signalling. Nature (2015) 526(7575):666-71. doi: 10.1038/nature15541

27. Man SM, Karki R, Kanneganti TD. Molecular Mechanisms and Functions of Pyroptosis, Inflammatory Caspases and Inflammasomes in Infectious Diseases. Immunol Rev (2017) 277(1):61-75. doi: 10.1111/imr.12534

28. Kayagaki N, Wong MT, Stowe IB, Ramani SR, Gonzalez LC, AkashiTakamura S, et al. Noncanonical Inflammasome Activation by Intracellular
LPS Independent of TLR4. Science (2013) 341(6151):1246-9. doi: 10.1126/ science. 1240248

29. Franchi L, Eigenbrod T, Munoz-Planillo R, Ozkurede U, Kim YG, Arindam C, et al. Cytosolic Double-Stranded RNA Activates the NLRP3 Inflammasome via MAVS-Induced Membrane Permeabilization and K+ Efflux. J Immunol (2014) 193(8):4214-22. doi: 10.4049/jimmunol.1400582

30. Vigano E, Mortellaro A. Caspase-11: The Driving Factor for Noncanonical Inflammasomes. Eur J Immunol (2013) 43(9):2240-5. doi: 10.1002/ eji.201343800

31. Alexopoulou L, Holt AC, Medzhitov R, Flavell RA. Recognition of DoubleStranded RNA and Activation of NF-kappaB by Toll-Like Receptor 3. Nature (2001) 413(6857):732-8. doi: 10.1038/35099560

32. Wypych TP, Wickramasinghe LC, Marsland BJ. The Influence of the Microbiome on Respiratory Health. Nat Immunol (2019) 20(10):1279-90. doi: 10.1038/s41590-019-0451-9

33. van Ruissen MCE, Bos LD, Dickson RP, Dondorp AM, Schultsz C, Schultz MJ. Manipulation of the Microbiome in Critical Illness-Probiotics as a Preventive Measure Against Ventilator-Associated Pneumonia. Intensive Care Med Exp (2019) 7(Suppl 1):37. doi: 10.1186/s40635-019-0238-1

34. Chellappan DK, Sze Ning QL, Su Min SK, Bin SY, Chern PJ, Shi TP, et al. Interactions Between Microbiome and Lungs: Paving New Paths for Microbiome Based Bio-Engineered Drug Delivery Systems in Chronic Respiratory Diseases. Chem Biol Interact (2019) 310:108732. doi: 10.1016/ j.cbi.2019.108732

35. Wang J, Sahoo M, Lantier L, Warawa J, Cordero H, Deobald K, et al. Caspase11-Dependent Pyroptosis of Lung Epithelial Cells Protects From Melioidosis While Caspase-1 Mediates Macrophage Pyroptosis and Production of IL-18. PLoS Pathog (2018) 14(5):e1007105. doi: 10.1371/journal.ppat.1007105

36. Deng M, Tang Y, Li W, Wang X, Zhang R, Zhang X, et al. The Endotoxin Delivery Protein HMGB1 Mediates Caspase-11-Dependent Lethality in Sepsis. Immunity (2018) 49(4):740-53.e7. doi: 10.1016/j.immuni.2018.08.016

37. Chen R, Zeng L, Zhu S, Liu J, Zeh HJ, Kroemer G, et al. cAMP Metabolism Controls Caspase-11 Inflammasome Activation and Pyroptosis in Sepsis. Sci $A d v$ (2019) 5(5):eaav5562. doi: 10.1126/sciadv.aav5562

38. Wang YC, Liu QX, Zheng Q, Liu T, Xu XE, Liu XH, et al. Dihydromyricetin Alleviates Sepsis-Induced Acute Lung Injury Through Inhibiting NLRP3 Inflammasome-Dependent Pyroptosis in Mice Model. Inflammation (2019) 42(4):1301-10. doi: 10.1007/s10753-019-00990-7

39. Wu J, Yan Z, Schwartz DE, Yu J, Malik AB, Hu G. Activation of NLRP3 Inflammasome in Alveolar Macrophages Contributes to Mechanical StretchInduced Lung Inflammation and Injury. J Immunol (2013) 190(7):3590-9. doi: $10.4049 /$ jimmunol.1200860

Conflict of Interest: The authors declare that the research was conducted in the absence of any commercial or financial relationships that could be construed as a potential conflict of interest.

Copyright $\odot 2021$ Jin, Ding, Yang, Li, Deng, Liao, Lv, Pitt, Billiar, Zhang and Li. This is an open-access article distributed under the terms of the Creative Commons Attribution License (CC BY). The use, distribution or reproduction in other forums is permitted, provided the original author $(s)$ and the copyright owner(s) are credited and that the original publication in this journal is cited, in accordance with accepted academic practice. No use, distribution or reproduction is permitted which does not comply with these terms. 